\title{
An Empirical Research on Service-Oriented Architecture (SOA) for Data Exchange in BIGDATA Systems
}

\section{T. Lavanya}

\begin{abstract}
Service-Oriented Architecture is a method for scheming dealing and organizing systems that represent ecofriendly business functionality. The objective of this study is to find out the critical success factors that need to implement SOA in BIG DATA systems. Our study aimed at classifying these erroneous performs in execution of SOA. The acceptance of SOA has interested creators to text its requests and applications. The analysed results would be very useful for researchers who would like to implement SOA with BIG DATA systems. SOA lead numerous advantages such as value-added flexibility and appropriate alignment among processes as well as reduced cost of integration and maintenance. Generally, BIG DATA anxieties large-volume, composite, rising figures groups with numerous, self-directed sources. BIG DATA claims where data gathering has grownup extremely and is elsewhere the aptitude of usually used software utensils to detention, accomplish and development within the rise [1]. The greatest essential task for the BIG DATA applications is to discover the large volumes of data and excerpt valuable material or information for upcoming actions. The main purpose of this study is to identify the important factors that are needed to implement SOA in BIG DATA systems. Zhang and Yang suggests a reengineering approach which will restructure the legacy systems that leads to SOA by considering of an organization. This paper also express various challenges of $\mathrm{SOA}$ and identify the problems that improve SOA based services for data exchange in BIG DATA systems.
\end{abstract}

Keywords: SOA, BIG DATA Systems (BDS), Legacy Systems, Web Services.

\section{INTRODUCTION}

The Service-Oriented Architecture (SOA) is an improved approach which will solve the issues in legacy or old systems and handles huge volumes of data such as BIGDATA Systems. SOA lead numerous advantages such as value-added flexibility and appropriate as well as reduced cost of integration and maintenance. SOA is a method for manipulative dealing and positioning schemes that signify refillable functionality. SOA delivers keys for mixing miscellaneous organizations that sustenance interoperability, movable link and reclaim. SOA is self-possessed of loosely coupled, discoverable, ecological and interoperable services. Web Services are used to implement the SOA in which service interface describes using the web service description languages. It comprises of a set of components as services that can be invoked and whose are published and discovered $[2,3]$, with the development of Big Data, their application in service started increasing the number of services on the internet. As a consequence, service-relevant data become too big to be professionally preserved by out-dated

Revised Manuscript Received on January 2, 2020.

T.Lavanya, Head, Department of Computer Science, Govt. Arts College for Women, Krishnagiri, Tamilnadu, India, E-mail klavan34@gmail.com approaches. SOA, there is a motionless a lack of experiential indication for factors implementing BIG DATA systems. This study showed that is easy to understand at the first glance that SOA capabilities will prepare better infrastructure than systems and needs which can drive technology towards the organizational goals.

\section{SERVICE-ORIENTED ARCHITECTURE}

Services are supposed to be designed for achieving main goals of SOA viz. reusability, interoperability, increasing organizational agility etc., SOA submissions could also be established which would mix various structures essential for SOA implementations. A number of groups which assuming SOA and optional four areas of acceptance tasks: program controlling, association, knowledge and supremacy. Wang (2013) assumed the SOA for the investigation and enterprise of business developments such as facility identification, requirement and understanding. SOA edition allows the creator to stunned many dispersed contests such as contract management, safety policies, claim integration etc., SOA support Service-Orientation principles and hence interaction between services much easier. We use SOA for establishing and exploiting spread competences of diverse domains and the technology that they use. In SOA, service identified by an interface and it makes service as a platform independent. Implementation of SOA is done by the web services and is dynamic in nature, which is having adaptive in nature such as services can add new service, integrate and remove old services. There is inaccessibility of service assembly and basis code for services. It makes problematic to test SOA based applications. This paper suggests service assessment measures serve as a position for both service provider and builder to evaluate the test support of SOA software.

SOA is needed because the modern enterprise deals with complex systems with fast growing technological changes, whereas these systems are loosely coupled between services and service consumers. The migration strategy of SOA is required minimize cost, increase network security, reduce data redundancy, gain transparency and reasonability of services from the legacy systems. The main goal of SOA adoption improving processes in an integrated environment within and outside the organization. During the integration process, some of the services or components may not be reused due to the technical constraints or incompatibility with the operating system of the legacy systems.

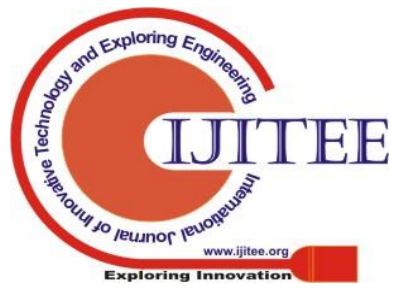


It also helps to improve visibility control of SOA in order to minimize risk. This research aims to lean-to light on the area of SOA with BIG DATA systems from the viewpoint of structural needs.

SOA is a method for organizations growth and incorporation in which functionality is assembled around corporate developments and wrapped as interoperable facilities. SOA also defines IT arrangement that allows dissimilar applications to argument data with one another while they contribute in business processes. An SOA aims to insecurely pair services with operating systems, software design languages, and other technologies that inspire applications. This process is very similar to what happened with equipment while it evolved. SOA splits functions into dissimilar units, or amenities, which are made available over a web so that the applications can trust and reclaim those functions. SOA notions are built upon mature ideas of disseminated figuring and segmental programming that progression in various mechanical infrastructure machineries and general software engineering have made possible. Operations can use one or more of these protocols, might use a file-system device to connect data following an express edge description between courses compatible to the SOA concept. Service-oriented architecture (SOA) is supposed to be the well-organized mixing thought to overwhelm the difficulties of construction professional systems. The benefit obtainable by SOA is functionality reclaim through service allowed applications. Although SOA offerings many talented lead for assimilating legacy systems, many topics need to be determined.

\section{BIG DATA SYSTEMS (BDS)}

BIG DATA Systems help to create business value by means of increased velocity and agility, improved reliability and support as well as enhanced cost efficiency. Generally, BIG DATA anxieties large-volume, composite, is raising data sets with manifold, self-directed causes. Big Data requests where data gathering has grownup extremely and is elsewhere the ability of usually used software tools to detention, accomplish and development within and the most fundamental contests for the Big Data applications is to discover the large volumes and data and abstract useful data or familiarity for future actions[4].

SOA is greatly changing the way that IT vendors use BIG DATA applications because all the broken part in the legacy systems are considered as layers that consists of services and components to be reused. Furthermore, the design and architectural model need to be built-in so that the technical gap is a narrowed to help vendors to minimize the implementation issues, in particular BIG DATA Systems. In terms of enterprises, it creates reusable network with interoperable services which is really very helpful for vendors in implementing these systems. This study has been conducted to share ideas about the factors of SOA to implement BIG DATA Systems. The factors such as SOA migration strategy of legacy systems and also played a vital role which has been revealed through empirical evidence [5]. SOA permits businesses to rapidly respond to trends in the current market in a cost-efficient manner and improves the systems. The analysed results would be very useful for academicians and researchers who would like to write articles in terms of implement SOA with BIG DATA Systems.

The statistics growing unaccompanied would be wonderful for an honestly active distribution. So, here we have a Big Data problem, anxieties conscious data. So to essentially fully screen all the data that permits over the system that is Big Data ready. SOA and Big Data as they are basically tangled. Data services spread the value of corporate services in a SOA. Data satisfied and contact is reliable and trustworthy. It eliminates the complications of retrieving varied data. SOA potentials to shorten challenging ultimately. Services are self-directed, displaced, with fully known boundaries, and distinct from the crosscutting anxieties of the application, the service can be verified as a 'black box' using present a stub corresponding to the service it calls. Data services diminish hazard and idleness in the expertise landscape. SOA authority delivers precious utensils with the recording of relations and needs of services, progressions, strategies and uses.

\section{LEGACY SYSTEMS\& RESULTS}

During the integration process, some of the services or components may not be reused due to the technical constraints or incompatibility with the operating system of the legacy system. The SOA is an improved approach which will solve issues in legacy or old systems and handles huge volumes of data. SOA really serves both the purpose of organizational needs such as cross-functional communication and integration with the legacy system. The highlight issues in process requirements, architecture and design in legacy systems. The organizations have to keep original data and functionality while it transforms to new system for legacy system. SOA provide a competitive edge among its competitors and at the same time provide single integrated reusable services from the legacy systems to avoid long implementation delays and reduce maintenance cost. The legacy systems are applications which are based on organizations has to keep original data and functionality while it transforms to new system from the legacy because it is very important as it has the organizations information flow. In addition, legacy systems can be better function together with the SOA. Moreover, technical progressions and variations in usability also inspire to deed new structures and include them in legacy systems to web services [6]. Our legacy systems are migrated to web services, and then through composition of web services, interplay of web services is feasible. The key solution suggested her is keeping functionality in the same legacy system. Enfolding practice is planned to brand work together functionalities of legacy systems nearby as web services.

Legacy systems typically have exclusive data characterizations. This repeatedly makes the semantic inconsistency among them and extra solicitations. The cavity concerning the exclusive data definition and SOA semantic substance desires to be associated. 
This paper clarifies how the concerns are determined in order to assimilate heritage systems within the SOA. It reuses and can also streamline interconnection to and convention of legacy systems. SOA that summarizes a business-oriented arrangement and has also been demonstrated several of the tactics, such schemes can place themselves to the reputation of well-defines, extremely inter-operable interfaces. Legacy classifications necessity to initiative SOA acceptance to the inordinate worth and deliver the most value-generating processes, because they were automatic than the other contemporary systems. The lead of legacy systems actual because systems are more specialists for applying value in the automation initiatives without incorporating the legacy systems is bound to have limited success. Legacy systems that started as the focus areas for their respective projects lost their appeal as sources of continued system development.

\section{WEB SERVICES}

Web Services can be used as refillable mechanisms that support interoperable machine-to-machine interface over the system. The web service is a typical that is the favoured way to understand SOA. Realizing SOA using web services can be trained as edition of dispersed constituent expertise with web organization. Some of the significant benefits of using web services as the skill platform for applying SOA are derivative from the way in which the WWW achieved its wonderful success. Suppleness and extensibility of web services permits various communication discussion designs to be applied for various types of clients. Web services are intended around the displaced argument of messages. Web service support open values which mark cross-platform use. Web services are designed for use between organizations and are firewall friendly. Web services have been measured as the capable knowledge to overawe the noninteroperability problem related with present handling systems. The SOA concept is often used as an equivalent to web services. The web services will be able to update selected fields not delete any data point. Each application has its own web service which is responsible to handle all requests related to that application. The web service using SOA doesn't only allow commercial process, but its loosely coupled environment also helps groups to assess their process and to put up fluctuations in process without affecting entire architecture. Web services are emerging as solution for the growth and disposition for actual mechanisation of inter-organizational interactions [7].

\section{CONCLUSIONS}

A SOA in which software mechanisms are unprotected as services on the network and can be reclaimed for dissimilar applications and for unlike purposes. The service component of web services refers to the conceptual idea behind web services. SOA emphases on distributing functionality as a set of dispersed services that can be arranged and bound at performance time. The experimental knowledge gained will be helpful in taking decisions for new problems in legacy systems. The implementation of this approach in legacy systems in different languages may bring out a lot of language-oriented facts and techniques which strengthen this process. The approach proposed in this work attempts to us SOA for effective migration from legacy systems to web services. This article has presented brief overviews, of the data services, through advanced are still evolving and their evolution toward more complex and sophisticated systems is estimated to endure for the predictable future. In this paper, we illustrated the features and the technology designed to support the effective cooperation of large scale enterprises. SOA will facilitate in near real-time capturing and processing of data and can share functionalities with these products. Comparative study in different perspectives may also be carried out in the legacy system of web services. The future work involves development of a legacy application of web services using SOA. Research chances of web services area are productive and significant for both instructors and experts. The main objective of this article is to propose a framework of web services and the SOA to be next generation infrastructure supporting decision-making.

\section{REFERENCES}

1. X.Wu,X.Zhu, G.Q.Wu and W.Ding, "Data Mining with big data", IEEE Trans. On Know/Data Eng., vol.26, no.1, pp, 97-107, Jan 2014.

2. L.rinivasan "An overview of SOA, Web Services and Grid Computing", HP white paper, Nov 2006.

3. M.P.Papazoglou and P.TraversO, "Service-Oriented Computing: state of the Art and Research challenges," IEEE computer society, Nov 2007.

4. A.Rajaraman and J.D.Ullman, "Mining of Massive Data Sets." Cambridge, U.K: Cambridge Univ. press2012.

5. Neaga,E.I and Hao.Y. "Towards BIGDATA Mining and Discovert, Proceedings of KIE conference: Research papers on knowledge, Innovation and Enterprise, London PP35-44, 2013.

6. Gerardo Canfora et al, "Migrating Interactive Legacy systems to web services", Proceedings of the conference on software maintenance and Reengineers (csmr'06) IEEE 2006.

7. Web Service Architecture requirements, W3C web services Architecture working Draft, Availabe online at http://WWW.W3.org/TR/2002/WD-WSA-reqs-2002/114.

\section{AUTHORS PROFILE}

T.Lavanya is presently working as Assistant Professor and Head, Dept. of Computer Science, Govt. Arts College for Women Krishnagiri, India. Her research interest includes Digital Electronics, Computer Architecture, Computer Networks, Web Services, Data Mining, Wireless Access Protocol, Advanced Operating Systems and Python Programming. She has twenty one years of teaching experience in the field of Information Technology and Computer Science. She has attended many conferences and several papers to her credit. Email: - klavan34@gmail.com 